\title{
An in vitro method to investigate microbicidal potential of human macrophages for use in psychosomatic research
}

Abbreviated running title: In vitro method for macrophage activity testing

Ulrike Kuebler $^{\mathrm{a}}$, PhD, Ulrike Ehlert ${ }^{\mathrm{a}}$, PhD, Claudia Zuccarella ${ }^{\mathrm{b}}$, M.S., Miho Sakai ${ }^{\mathrm{c}}, \mathrm{PhD}$, Andreas Stemmer ${ }^{\mathrm{c}}$, PhD, \& Petra. H. Wirtz ${ }^{\mathrm{b}}, \mathrm{PhD}$

${ }^{a}$ Department of Clinical Psychology and Psychotherapy, University of Zurich, Binzmühlestrasse 14, 8050 Zurich Switzerland.

${ }^{b}$ Biological and Health Psychology, University of Bern, Alpeneggstrasse 22, 3012 Bern, Switzerland. ${ }^{c}$ Nanotechnology Group, Swiss Federal Institute of Technology Zurich, Tannenstrasse 3, 8092 Zurich, Switzerland.

Conflicts of Interest and Source of Funding:

None declared. Chocosuisse Foundation Grant 2009 (to PHW) and Swiss National Science Foundation Grant PP00P1_128565/1 (to PHW)

\begin{abstract}
word count: 248; total text word count: 5498 (without references) or 6268 with references; number of figures: 2 , number of tables: 1
\end{abstract}

Corresponding author:

Petra H. Wirtz, Ph.D., University of Bern, Department of Psychology, Biological and Health Psychology, Alpeneggstrasse 22, 3012 Bern, Switzerland, E-mail address: petra.wirtz@psy.unibe.ch; Phone: +41 31631 5790; Fax: +41 316314155 


\begin{abstract}
Objective: Psychological states relate to changes in circulating immune cells, but associations with immune cells in peripheral tissues such as macrophages have hardly been investigated yet. Here, we aimed to implement and validate a method for measuring the microbicidal potential of ex vivo isolated human monocyte-derived macrophages (HMDM) as an indicator of macrophage activation.
\end{abstract}

Methods: The method was implemented and validated for two blood sampling procedures (short-term cannula insertion vs. long-term catheter insertion) in 79 participants (34 women, 45 men) aged between 18 and 75 yrs. The method principle is based on the reduction of 2-(4iodophenyl)-3-(4-nitrophenyl)-5-(2,4-dis-ulfophenyl)-2H tetrazolium, monosodium salt (WST-1) by superoxide anions, the first in a series of pathogen-killing reactive oxygen species produced by phorbol-myristate-acetate (PMA)-activated HMDM. Cytochrome c reduction and current generation were measured as reference methods for validation purposes. We further evaluated whether depressive symptom severity (Beck Depression Inventory [BDI]) and chronic stress (Chronic-Stress-Screening-Scale [CSSS]) were associated with macrophage microbicidal potential.

Results: The assay induced superoxide anion responses by HMDM in all participants. Assay results depended on blood sampling procedure (cannula vs. catheter insertion). Inter-assay variability as a measure for assay reliability was $\leq 10.92 \%$. WST-1 reduction scores correlated strongly with results obtained by reference methods (cytochrome c: $r=.57, p=.026$; current generation: $r$ 's $\geq .47, p$ 's<.033) and with psychological factors (depressive symptom severity: $r=.35$ [cannula insertion] vs. $r=-.54$ [catheter insertion]; chronic stress: $r=.36$ [cannula insertion]; $p$ 's<.047). 
Conclusions: Our findings suggest that the implemented in vitro method investigates microbicidal potential of HMDM in a manner that is valid and sensitive to psychological measures.

Keywords: human macrophages, microbicidal potential, reactive oxygen species, in vitro method, depressive mood, chronic stress

\section{Acronyms used in the text:}

ANOVA = univariate analyses of variance; BDI = Beck Depression Inventory; BMI = Body mass index; CSSS = Chronic-Stress-Screening-Scale; $\mathrm{C}_{\mathrm{Max}}=$ maximum current value registered; $\mathrm{C}_{\text {Sum }}=$ the sum of all current values; FBS = fetal bovine serum; HBSS = Hank's balanced salt solution; HMDM = human monocyte-derived macrophages; IFN- $\gamma=$ Interferon$\gamma ;$ LPS = Lipopolysaccharide; M1 macrophage = classically activated / inflammatory macrophage ; NADPH oxidase $=$ nicotinamide adenine dinucleotide phosphate oxidase; $\mathrm{OD}=$ optical densitiy; PBMCs_= peripheral blood nuclear cells; PBS = phosphate buffered saline; PMA = phorbol-myristate-acetate; ROS $=$ reactive oxygen species; $\mathrm{SDC}=$ Supplemental Digital Content; TNF- $\alpha=$ tumor necrosis factor- $\alpha$; WST-1 = 2-(4-iodophenyl)-3-(4nitrophenyl)-5-(2,4-dis-ulfophenyl)-2H tetrazolium, monosodium salt 


\section{Introduction}

A growing body of psychosomatic research documents that psychological states are linked to quantitative and qualitative alterations in circulating immune cells (e.g. (1, 2)). In contrast, relatively little is known about associations between psychological states and alterations in immune cells in peripheral tissues such as macrophages (mature, tissue-differentiated monocytes). Activated macrophages are important effector cells involved in warding off microorganisms and regulating inflammation (3). We recently found first evidence that acute stress relates to alterations in wound-induced macrophage activation but associations with longer-lasting psychological states without prior wound-induction have not yet been investigated (4). The lack of research in this field may be related to both the absence of and familiarity with methods to analyze macrophage activity. Given that macrophages are primarily activated to develop microbicidal effector functions (5), the purpose of our study was to systematically validate an in vitro method suitable for assessing microbicidal potential of human macrophages which may be used in future psychosomatic and psychobiological research.

Microbicidal effectiveness of human macrophages is due in large part to the production of reactive oxygen species (ROS) (6). ROS production is mediated by activation of the membrane-bound enzyme complex nicotinamide adenine dinucleotide phosphate (NADPH) oxidase. Once activated, NADPH oxidase transfers electrons from NADPH in the cytosol to extracellular or intraphagolysosomal oxygen molecules. These oxygen molecules are then chemically reduced to superoxide anions, a type of ROS (6). The superoxide anions formed in this reaction serve as precursors to other, more reactive ROS such as hydrogen peroxide and hypochlorous acid (7). The assay presented here to study the microbicidal potential of human macrophages is an adaptation of a method used in a permanent monocytic cell line (THP-1 cells) as described by Sakai et al. (8). To allow exploration of associations with psychological 
states, we adapted the existing method to ex vivo isolated human monocyte-derived macrophages.

The assay principle is based on the chemical reduction of the cell-impermeative tetrazolium salt WST-1 (2-(4-Iodophenyl)-3-(4-nitrophenyl)-5-(2,4-disulfophenyl)-2H-tetrazolium monosodium salt) by superoxide anions that are produced by phorbol myristate acetate (PMA)-activated human monocyte-derived macrophages (HMDM). HMDM represent the classically activated (M1) macrophage also known as the inflammatory macrophage phenotype (9). More specifically, human blood monocytes are isolated from whole blood and then differentiated in vitro into inflammatory macrophages. Inflammatory macrophages are characterized by high cytosolic expression of NADPH oxidase, the prerequisite for high oxygen-dependent microbicidal activity (10). To induce macrophage microbicidal activity intended to destroy pathogens, NADPH oxidase needs to be activated. Activation of NADPH oxidase can be experimentally induced by in vitro incubation with PMA (for details see (11)). Once activated, the NADPH oxidase catalyzes the reduction of oxygen to superoxide anions which in turn reduce WST-1 $(8,12)$. The reduction of WST-1 results in the formation of a colored, water-soluble formazan salt with increased absorbance at $450 \mathrm{~nm}(8,12)$. Consequently, the colorimetric measurement of formazan formation in the medium in which macrophages are suspended indicates superoxide anion-induced WST-1 reduction and thus macrophage (NADPH oxidase-mediated) microbicidal potential.

In the present study, the assay implementation and validation process was as follows: First, we tested the influence of various stimulation agents on superoxide anion production from human inflammatory macrophages as well as the influence of different cell numbers on WST1 reduction scores in pilot studies. Second, to ensure assay reliability, we investigated the inter-assay variability in a sample of men and women. Additionally, to ensure validity, we conducted the assay and compared results with reduced cytochrome c as a reference method. 
Next, in order to investigate sensitivity of the WST-1 macrophage assay to psychological states, we tested for associations of macrophage microbicidal potential with self-reported chronic stress and depressive symptoms severity. Notably, all blood samples for performing the WST-1 macrophage assay were collected by short-term cannula insertion (cannula group).

Since we recently found first indications that the blood sampling procedure (i.e. short-term cannula insertion or long-term venous catheter insertion) seems to affect macrophage microbicidal potential (4), we additionally aimed to compare WST-1 reduction scores based on blood samples obtained by short-term cannula insertion with WST-1 reduction scores based on blood samples obtained by venous catheter inserted long enough to start wound healing processes (13). For this purpose, in a third sample of healthy men blood samples were collected via an indwelling venous catheter inserted 2 hours before the WST-1 macrophage assay was performed (catheter group). We additionally validated the WST-1 macrophage assay in the catheter group by comparing WST-1 reduction scores with current generation as a reference method, and tested for associations between the WST-1 macrophage assay and depressive symptom severity.

\section{Materials and methods}

\section{$\underline{2.1 \text { Reagents and chemicals }}$}

We used the following reagents: Ficoll-Paque PLUS (Ficoll; no. 17-1440-02; GE Healthcare; Uppsala, Sweden); 2-(4-Iodophenyl)-3-(4-nitrophenyl)-5-(2,4-disulfophenyl)-2H-tetrazolium (WST-1; no. 150849-52-8, Dojindo Laboratories; Kumamoto, Japan); Interferon- $\gamma$ (IFN- $\gamma$; no. PHC4031, Invitrogen; Basel, Switzerland), tumor necrosis factor- $\alpha$ (TNF- $\alpha$; no. PHC3016, Invitrogen; Basel, Switzerland); Hank’s balanced salt solution without phenol red (HBSS; no. 14025050, Invitrogen; Basel, Switzerland); fetal bovine serum (FBS; no. 10106-169, 
Invitrogen; Basel, Switzerland); Lipopolysaccharide (LPS; no.L6529, Sigma-Aldrich; Buchs, Switzerland); phosphate buffered saline (PBS; no. P5368, Sigma-Aldrich; Buchs, Switzerland); phorbol 12-myristate 13-acetate (PMA; no. P8139, Sigma-Aldrich; Buchs, Switzerland); cytochrome c (no. C2037, Sigma-Aldrich; Buchs, Switzerland); RPMI-1640 Media with Glutamax (RPMI-1640; no. W9925E, Fisher Scientifc; Wohlen, Switzerland); Diff-Quick (no. 130832; Siemens, Zurich, Switzerland).

\section{$\underline{2.2 \text { Participants }}$}

The assay validation study was performed in three different samples and was conducted from January 2010 until March 2013.

\subsubsection{Study sample 1}

Study sample 1a consisted of 11 apparently healthy Caucasian adults (6 women, 5 men) aged between 23 and 34 yrs (mean age \pm SEM; $27 \pm 1.2$ yrs) who provided one blood sample each. Study sample $1 \mathrm{~b}$ consisted of four apparently healthy Caucasian women. Analogous to the subsequent experiments described below, we distinguished between blood sampling procedures: While blood samples were taken by short-term cannula insertion (cannula group) in study sample 1a, in study sample 1b blood samples were taken by an indwelling venous catheter inserted two hours before blood sampling (catheter group). The blood samples of study sample 1b were provided on six different days with assays run in triplicates on three days rendering a total of 12 measurements. 
The study sample consisted of 40 Caucasian adults (24 women, 16 men) aged between 18 and 75 yrs (mean age \pm SEM; $40 \pm 3.1$ yrs). Table 1 provides characteristics of this study sample. While female subjects were recruited via advertisements from the Canton of Zurich as part of an ongoing study on the effects of psychological stress on health outcomes, male subjects were recruited via advertisements from the Canton of Bern as part of an ongoing study assessing psychobiological mechanisms in hypertension and coronary heart disease. No specific exclusion criteria were applied.

Each participant provided a blood sample taken by short-term cannula insertion and completed the Beck Depression Inventory (BDI) as well as the Chronic Stress Screening Scale (CSSS) (see below). The study protocol was formally approved by the Ethics Committee of the University of Zurich, Switzerland, and by the Ethics Committee of the Canton of Bern, Switzerland. All subjects provided written informed consent.

\subsubsection{Study sample 3 (catheter group)}

We recruited 24 healthy, medication-free, non-smoking Caucasian men aged between 20 and 48 yrs (mean age \pm SEM; $37 \pm 1.5$ yrs). Subjects' characteristics are depicted in Table 1 . Study participants were in good physical and mental health as confirmed by telephone interview. Explicit exclusion criteria were: regular strenuous exercise, smoking, alcohol and illicit drug abuse, any heart disease, varicosis or thrombotic diseases, elevated blood sugar and diabetes, elevated cholesterol, liver and renal diseases, chronic obstructive pulmonary disease, allergies and atopic diathesis, rheumatic diseases, and current infectious diseases. If the personal or medication history was not conclusive, the participants' primary care physician was contacted for verification. 
Participants were recruited via advertisements and with the help of the Swiss Red Cross of the canton of Zurich. Each participant provided a blood sample taken at approximately 1:30 p.m. by an indwelling venous catheter inserted two hours before blood sampling and completed the BDI (see below). Notably, we previously used this catheter-insertion procedure as an openwound paradigm to preactivate monocytes (4). The study protocol was formally approved by the Ethics Committee of the Canton of Zurich, Switzerland, and written informed consent was obtained from all participants.

2.3 The WST-1 macrophage assay, an in vitro method for assessing macrophage NADPH $\underline{\text { oxidase-mediated microbicidal potential }}$

The method used in this study to measure macrophage superoxide anion production from ex vivo isolated human cells is an adaptation of a method used in a permanent monocytic cell line (THP-1 cells) as described by Sakai et al. (8).

\subsubsection{Monocyte isolation by adherence after Ficoll purification of peripheral blood nuclear} cells (PBMCs)

In order to separate the cells of interest, nine milliliters of blood were collected in EDTAcoated tubes (Sarstedt, Numbrecht, Germany), immediately layered on top of $10 \mathrm{ml}$ Ficoll (density-based cell separation medium) and centrifuged for 20 minutes at $300 \mathrm{~g}$ and $20^{\circ} \mathrm{C}$. After centrifugation, peripheral blood nuclear cells (PBMCs, i.e. lymphocytes and monocytes), were removed from the interface, washed twice in RPMI1640 medium, counted with a hematologic analyzer (KX-21N; Sysmex Digitana AG), and re-suspended to a concentration of $2.5 \times 10^{6} / \mathrm{ml}$ with RPMI1640 media supplemented with $10 \%$ FBS. Then, PBMC suspension aliquots of $1 \mathrm{ml}$ were transferred to 24-well cell culture plates (no. 4609; Semadeni; Ostermundigen, Switzerland). After incubation for $1 \mathrm{~h}$ at $37^{\circ} \mathrm{C}$ and $5 \% \mathrm{CO}_{2}$, the 
supernatant was discarded and the plate surface was rinsed five times with $1 \mathrm{ml}$ of warm $\left(25^{\circ} \mathrm{C}\right)$ 0.01M PBS to remove non-adherent PBMC, while monocytes remained adherent to the bottom of the plates. This monocyte isolation method is a well-established procedure to yield monocyte cultures of more than 90\% purity (14-18).

\subsubsection{Differentiation of human monocytes into macrophages and their separation}

The resulting adherent monocyte layer (obtained as described above) was diluted with $1 \mathrm{ml}$ RPMI1640 media supplemented with 10\% FBS. Subsequently, we added $2 \mu$ IFN- $\gamma, 2 \mu 1$ TNF- $\alpha$, and $0.5 \mu 1$ LPS resulting in a final concentration of $20 \mathrm{ng} / \mathrm{ml} \mathrm{IFN-} \gamma, 20 \mathrm{ng} / \mathrm{ml}$ TNF- $\alpha$, and $300 \mathrm{ng} / \mathrm{ml}$ LPS to promote differentiation of monocytes into inflammatory macrophages $(3,19,20)$. After incubation for $48 \mathrm{~h}$ at $37^{\circ} \mathrm{C}$ and $5 \% \mathrm{CO}_{2}$, the supernatant was discarded and the adherent macrophage layer was washed three times with $1 \mathrm{ml}$ of warm $\left(25^{\circ} \mathrm{C}\right) 0.01 \mathrm{M}$ PBS to remove traces of culture media and non-adherent cells.

\subsubsection{WST-1 assay to determine macrophage superoxide anion production}

Next, the resulting macrophage monolayer (obtained as described above) was overlaid with 1 ml HBSS buffer solution. Subsequently, $2 \mu 1$ WST-1, $0.5 \mu$ LPS, $2 \mu 1$ IFN- $\gamma, 2 \mu 1$ TNF- $\alpha$, and $0,5 \mu 1$ PMA were added, resulting in a final concentration of $100 \mu \mathrm{M}$ WST-1, $300 \mathrm{ng} / \mathrm{ml} \mathrm{LPS}$, $20 \mathrm{ng} / \mathrm{ml} \mathrm{IFN-} \gamma, 20 \mathrm{ng} / \mathrm{ml} \mathrm{TNF-} \alpha$, and $50 \mathrm{nM}$ PMA. This was followed by an incubation period of 4 hours at $37^{\circ} \mathrm{C}$ and $5 \% \mathrm{CO}_{2}$. Then, the supernatant was removed and used to determine WST-1 reduction by reading the absorbance with a spectrophotometer (SmartSpec Plus, Bio-Rad Laboratories, Inc., Hemel Hempstead, United Kingdom for study samples 1 and 3; Synergy HT, BioTek, Luzern, Switzerland, for study sample 2) at $450 \mathrm{~nm}$ against water 
as blank. Higher optical densities (ODs) as obtained by absorbance reading are associated with higher amounts of WST-1 reduction and thus of superoxide anions generated by HMDM.

\section{$\underline{2.4 \text { Assay implementation and validation procedures }}$}

\subsubsection{Identification and verification of macrophage superoxide anion production stimulating} agents (study sample 1)

In order to verify the applicability of the cell-line tested in vitro method for ex vivo isolated HMDM, we tested the influence of different stimuli on the superoxide anion production of ex vivo isolated human monocytes/macrophages. Following Sakai et al (8), we used stimuli which initiate either cell differentiation, cell activation or both cell differentiation and activation. For cell differentiation, i.e. differentiation of monocytes into inflammatory macrophages, we used the combination of LPS, IFN- $\gamma$, and TNF- $\alpha$; for cell activation, i.e. activation of the NADPH oxidase, we used PMA. Combined differentiation and activation included the use of LPS, IFN- $\gamma$, TNF- $\alpha$, and PMA (detailed protocol in Section 2.3). The stimulation experiments were performed in the 12 subjects of study sample 1a (cannula group) and 12 blood samples of study sample 1b. One female subject of study sample $1 \mathrm{a}$, however, had to be excluded due to problems with venipuncture rendering a final study sample of 11 (6 women, 5 men) for stimuli testing.

\subsubsection{Cell-number dependent WST-1 reduction (study sample 2, “cannula group”)}

In order to obtain pilot data regarding the minimum cell concentration of PBMCs needed to induce measurable PMA-induced superoxide anion release by HMDM we performed the 
WST-1 macrophage assay using five different PBMC concentrations: 3.0, 2.5, 2.0, 1.5, and $1.0 \times 10^{6}$ PBMCs. Experiments were carried out in cells obtained from five female subjects of study sample 2 .

\subsubsection{Determination of assay reliability (study sample 2, "cannula group”)}

Assay reliability was assessed by testing inter-assay variability of blood samples split either before or after Ficoll purification. For details on reliability testing see Supplemental Digital Content (SDC) 1. Assay reliability was tested in 24 subjects of study sample 2. One female subject, however, had to be excluded due to technical problems rendering a final study sample of 23 (18 women, 5 men) for reliability testing.

\subsubsection{Assay validation by comparison with reference method}

2.4.4.1 Validation of the WST-1 macrophage assay by cytochrome c (study sample 2, “cannula group”)

Like WST-1, cytochrome c is reduced by superoxide anions (21) and can therefore be used analogous to WST-1 as a detector for superoxide anion production. We thus measured the amount of reduced cytochrome c to validate the WST-1 macrophage assay. For details on this reference method see SDC 2. Assay validation using cytochrome c as reference method was performed in the first 15 participants of study sample 2 (11 women, 4 men).

2.4.4.2 Validation of macrophage superoxide anion production by generation of electrical current (study sample 3, “catheter group”) 
Current generation observed in the biofuel cell developed by Sakai et al. (8) primarily originates from PMA-induced superoxide anion release by THP-1-derived macrophages. The greater superoxide anion release, the greater current generation. Therefore, the quantity of electrical power produced in this biofuel cell setup represents a suitable criterion for the validation of the WST-1 macrophage assay. For details on this reference method see SDC 3. Assay validation with this reference method was carried out in 21 subjects of study sample 3.

\subsubsection{Macrophage number corrected WST-1 reduction (study sample 2, "cannula group")}

Our WST-1 assay principle is based on monocytes included in $2.5 \times 10^{6}$ PBMC that we stimulate by incubation with different agents to differentiate into macrophages. Thus, possible differences in WST-reduction scores may relate to differences in the number of macrophages derived from that same number of PBMCs. Notably, the same number of PBMCs may result in different numbers of adherent monocyte-derived macrophages. Consequently, we aimed to determine the number of adherent cells per well as an indicator of the final macrophage number per well. We corrected WST-1 reduction score per 2.5 x $10^{6}$ PBMC (i.e. total WST-1 reduction scores) by post-hoc counted macrophage numbers to obtain a second measure of WST-1 reduction, i.e. macrophage number corrected WST-1 reduction score (corrected WST1 reduction score). For details on the cell-counting method see SDC 4. The number of adherent cells was determined in the first 30 participants of study sample 2 (15 women, 15 men).

\section{$\underline{2.5 \text { Psychological assessment }}$}


To test for associations between microbicidal potential and psychological factors, the validated German versions of the following self-report questionnaires were used.

\subsubsection{Depressive symptom severity (study sample 2 and study sample 3)}

Depressive symptom severity was assessed with the 21-item Beck Depression Inventory (BDI), where scores $\geq 10$ indicate possible clinical depression (22). The BDI was developed for the assessment of depressive symptoms that correspond to the Diagnostic and Statistical Manual of Mental Disorders-IV criteria for major depressive disorders and measures a

somatic and a cognitive-affective dimension of depression (22). The BDI assesses the frequency and/or severity of symptoms related to sadness, feelings of guilt, perceptions of self-worth, suicidal ideation, and changes in appetite and body weight, among other characteristics. Items have a 4-point scale ranging from 0 (symptom not present) to 3 (symptom very present). Higher scores mean higher depressive symptom severity.

\subsubsection{Chronic stress (study sample 2, “cannula group”)}

Chronic stress was measured using the 12-item Chronic Stress Screening Scale (CSSS) of a larger chronic stress questionnaire (23). The CSSS assesses the frequency of experiencing work overload (4 items), worries (4 items), lack of social recognition (2 item), excessive demands at work (1 item), and social overload (1 item). Items have a 5-point rating format reflecting frequency (ranging from 1 [never] to 5 [very often]). Higher scores mean higher chronic stress. 


\section{$\underline{2.7 \text { Statistical analysis }}$}

Data were analyzed using SPSS Inc. version 19.0 for Windows (Statistical Package for the Social Sciences, SPSS, Chicago, IL, USA) and presented as mean \pm SEM. All tests were 2tailed with the level of significance set at $p<.05$. Body mass index (BMI) was calculated by the formula weight in $\mathrm{kg} /\left(\right.$ height in $\mathrm{m}^{2}$. WST-1 scores were corrected for macrophage numbers: “corrected WST-1 reduction” refers to WST-1 reduction scores per 10000 macrophages.

Difference testing. We used univariate analyses of variance (ANOVAs) to test for differences (1) between cannula and catheter group (Table 1, group characteristics), (2) between men and women of the cannula group, (3) between the four stimulation conditions (testing of stimulating agents, study sample 1), and (4) between the five cell number conditions (cellnumber dependent WST-1 reduction, study sample 2).

Reliability testing. We determined assay reliability by calculating the mean percentage difference between samples A1 and B (i.e. before Ficoll purification) and between samples A1 and A2 (i.e. after Ficoll purification).

Validity testing. Pearson's correlations were used to validate superoxide anion induced macrophage WST-1 reduction against the reference methods, i.e. cytochrome c reduction and superoxide anion induced macrophage current generation. Since current generation was monitored continuously, we extracted two indices to reflect power generation from the fourhour recording period: first the maximum current value registered $\left(\mathrm{C}_{\mathrm{Max}}\right)$ and then the sum of all current values $\left(\mathrm{C}_{\text {Sum }}\right)$.

Association testing. To test whether psychological factors are associated with the WST-1 macrophage assay correlations were calculated between psychological factors (i.e. BDI and CSSS) and total or corrected WST-1 reduction scores adjusting for age, BMI, and gender 
(where applicable) as a priori selected control variables because of known alterations in a variety of immune functions with age, BMI and gender (24-26). Unadjusted correlations are presented as well.

\section{$\underline{3 \text { Results }}$}

\subsection{Group characteristics (study sample 2, study sample 3)}

Table 1 provides the characteristics of study sample 2 (cannula group; $n=40$ ) and study sample 3 (catheter group; $n=24$ ). The two groups did not significantly differ in terms of age and BMI. The catheter group had lower BDI scores than the cannula group and subgroup analysis restricted to men did not change this result ( $6.0 \pm 1.3$ [cannula group] vs. $3.2 \pm 0.7$ [catheter group]; $p=.044)$. In addition, total WST-1 reduction scores were higher in the catheter group than in the cannula group. Within in the cannula group, men and women did not significantly differ in total or corrected WST-1 reduction scores ( $p$ 's > .62) independent of age and BMI.

$\underline{3.2}$ WST-macrophage assay implementation and validation

\section{$\underline{\text { 3.2.1 Pilot data }}$}

3.2.1.1 Identification and verification of macrophage superoxide anion production $\underline{\text { stimulating agents (study samples } 1 a \text { and } 1 b \text { ) }}$

WST-1 reduction scores differed significantly between the four stimulation procedures (cannula group: $F(3,43)=12.78, p<.001, n=12$; catheter group: $F(3,47)=347.72, p<$ $.001, n=11$; Figure 1A). Post-hoc calculated univariate ANOVAs revealed that 
monocytes/macrophages exposed to both differentiation and activation agents showed the greatest WST-1 reduction capacity compared to cells treated with either none of the stimulation agents (cannula group: $F(1,21)=23.7, p<.001$; catheter group: $F(1,23)=$ 492.54, $p<.001$; negative control) or differentiation (cannula group: $F(1,21)=16.91, p=$ .001 ; catheter group: $F(1,23)=443.28, p<.001$; ) and activation (cannula group: $F(1,21)=$ 4.95, $p=.038$; catheter group: $F(1,23)=331.45, p<.001$; ) agents alone.

The amount of WST-1 reduction reflects NADPH oxidase-derived superoxide anion production by HMDM. These results indicate that stimulation by combined cell differentiating and activating agents constitutes the most effective procedure for inducing superoxide responses.

\subsubsection{Cell-number dependent WST-1 reduction (study sample 2, “cannula group”)}

Figure 1B shows PMA-induced superoxide anion production by macrophages with WST-1 reduction scores plotted against increasing concentrations of PBMCs. WST-1 reduction scores differed significantly over PBMC concentrations $(\mathrm{F}(3.7 / 14.9)=37.14, \mathrm{p}<.001, n=5)$.

\subsubsection{Assay reliability (study sample 2, "cannula group”)}

Reliability assessment based on inter-assay variability of blood samples split before Ficoll purification was $9.77 \%( \pm 6.91 \mathrm{SD}$, range $=0.00-21.65 \%, n=23)$. Inter-assay variability of blood samples split after Ficoll purification was $10.92 \%$ ( \pm 6.55 SD, range $=0.95-21.93 \%$, $n=23)$. 


\subsubsection{Assay validation by comparison with reference method}

3.2.3.1 Validation of the WST-1 macrophage assay by cytochrome c (study sample 2, “cannula group”)

Higher cytochrome c reduction scores correlated significantly with higher WST-1 reduction scores $(r=.57 ; p=.026 ; n=15 ;$ Figure 2A).

3.2.3.2 Validation of macrophage superoxide anion production by generation of electrical current (study sample 3, “catheter group”)

We observed current generation in HMDM of 21 participants $\left(\mathrm{C}_{\mathrm{Max}}=0.10 \pm 0.01 \mu \mathrm{A}\right.$, range $=$ 0.05-0.24 $\mu \mathrm{A} ; \mathrm{C}_{\text {Sum }}=284.12 \pm 34.83 \mu \mathrm{A}$, range $\left.=139.01-765.77 \mu \mathrm{A}\right)$. Higher WST-1 reduction scores correlated significantly with higher current generation ( $r$ (WST-1 reduction / $\left.\mathrm{C}_{\text {Max }}\right)=.48 ; p=.028$; Figure $\left.2 \mathrm{~B}\right) ; r\left(\right.$ WST-1 reduction $\left./ \mathrm{C}_{\text {Sum }}\right)=.47 ; p=.033$; Figure $\left.2 \mathrm{C}\right)$. Exclusion of two outlier subjects improved these positive associations ( $r$ (WST-1 reduction / $\left.\left.\mathrm{C}_{\text {Max }}\right)=.50 ; p=.030\right) ; r\left(\right.$ WST -1 reduction $\left.\left./ \mathrm{C}_{\text {Sum }}\right)=.52 ; p=.024\right)$.

3.2.4 Associations between macrophage-derived superoxide anion production and psychological factors

\subsubsection{Cannula group (study sample 2)}

In the cannula group ( $n=40)$, both higher chronic stress (i.e. CSSS scores) and higher depressive symptom severity (i.e. BDI scores) correlated significantly with higher WSTreduction scores $(r$ (CSSS / total WST-1 reduction $)=.36, p=.030 ; r(\mathrm{BDI} /$ total WST-1 
reduction) $=.35, p=.032$ ). Age, $\mathrm{BMI}$, and gender were controlled. Additional correction of total WST-1 reduction scores for macrophage number per well did not change results ( $r$ (BDI $/$ corrected WST-1 reduction $)=.39, p=.042, r($ CSSS $/$ corrected WST-1 reduction $)=.39, p$ $=.047)$. Without controlling for any confounders the observed association with chronic stress slightly increased $(r$ (CSSS / total WST-1 reduction $)=.38, p=.017)$ whereas the observed association with depressive symptom severity decreased $(r$ (BDI / total WST-1 reduction) $=$ $.24, p=.14)$.

\subsubsection{Catheter group (study sample 3)}

In the catheter group $(n=24)$, higher BDI scores correlated significantly with lower total WST-reduction scores measured from blood sampling obtained two hours after catheter insertion $(r$ (BDI / total WST-1 reduction $)=-.54, p=.010)$. Age and BMI were controlled. Without controlling for covariates results become of borderline significance ( $r$ (BDI / total WST -1 reduction) $=-.35, p=.098$ )

\section{Discussion}

In this study, we implemented and validated an in vitro method, that allows the investigation of NADPH oxidase-mediated microbicidal potential from ex vivo isolated HMDM.

In a pilot study, we verified applicability of the cell-line tested in vitro method for ex vivo isolated HMDM. Similar to THP-1 cells and independent of the blood sampling procedure, ex vivo isolated human monocytes/macrophages exposed to both differentiation and activation agents produced the greatest amounts of superoxide anions compared to cells treated with either differentiating or activating stimuli alone. Application of the WST-1 macrophage assay 
evoked significant PMA-induced superoxide anion responses by HMDM in all participants of study sample 2 (cannula group) and study sample 3 (catheter group). These findings indicate that the in vitro method described for THP-1 cells is applicable to ex vivo isolated HMDM. We further showed that higher depressive symptoms and higher chronic stress were associated with higher WST-1 reduction scores $(r \geq .35)$ in the cannula group. These data indicate that the WST-1 macrophage assay has utility for studies on effects of sustained psychological states on macrophage activity. Notably, the direction of these associations could be confirmed in men and women separately (data not shown).

Interestingly, comparable associations with psychological factors were obtained when the total WST-1 reduction scores were corrected for macrophage number per well (corrected WST-1 reduction scores). This suggests that determination of total WST-1 reduction scores seems to allow for adequate assessment of microbicidal potential per macrophage. However, it should be kept in mind that not the total WST-1 reduction scores, but rather the corrected WST-1 reduction scores reflect microbicidal potential per macrophage. Also, total WST-1 reduction scores reflect the microbicidal potential based on PBMC counts, i.e. the microbicidal potential of those macrophages differentiated from $2.5 \times 10^{6}$ PBMCs.

In contrast to the cannula group where positive associations between WST-1 reduction and depressive symptom severity were found, higher depressive symptom severity was associated with lower levels of WST-1 reduction $(r=-.54)$ in the catheter group. This opposite finding not only corroborates the data above indicating assay sensitivity to psychological factors, but also provides first indication that depending on the activation status of the macrophages (i.e. basal activity of in vivo unstimulated cells or reactive to in vivo stimulation by catheterinduced wound-application), psychological factors can be associated in a different way with microbicidal potential of macrophages. Given this reasoning, the observed opposite association with depressive symptoms are not contradictory but rather present different 
phenomena. Thus, basal macrophage activity might be higher with increasing depression scores whereas reactive macrophage activity at the same time seems to be blunted. A speculative explanation for the observed opposite association with depressive symptoms may relate to exhaustion of this inflammatory macrophage type (i.e. decreased reactivity to in vivo stimulation by wound-induction) due to chronically elevated basal activation. But it also cannot be ruled out that this observation relates to a potentially altered cell differentiation processes following wound-induction with depressive symptoms.

Furthermore, in a small subsample of study sample 2 (cannula group) we investigated PBMCnumber dependent WST-1 reduction and found an increase in superoxide anion production with increasing PBMC concentrations in the range between $1.0-3.0 \times 10^{6}$ PBMCs. However, the superoxide anion production of $1.0 \times 10^{6}$ PBMCs was quite low suggesting that the minimum cell concentration of PBMCs needed to induce measureable PMA-induced superoxide anion release by HMDM after blood sampling by short-term cannula insertion ranges between 1.0 and $1.5 \times 10^{6}$ PBMCs. Higher PBMC cell numbers than this minimum concentration are therefore recommended to allow for optimal detection. In a larger subsample of study sample 2 (cannula group) we also tested assay reliability by using two different indicators of inter-assay variability. Inter-assay variabilities of the WST-1 macrophage assay were $\leq 10.92 \%$ suggesting that the assay is sufficiently robust for quantitative research. Furthermore, we evaluated the validity of the assay by comparing WST1 reduction scores with reference methods and found that greater WST-1 reduction was associated with greater cytochrome c reduction (cannula group) or current generation (catheter group), respectively. The observed moderate heights of the correlation coefficients in our study $\left(r\right.$ (WST-1 reduction / cytochrome c reduction) $=.57 ; r$ (WST-1 reduction / $\left.\mathrm{C}_{\text {Max }}\right)$ $=.48 ; r\left(\right.$ WST -1 reduction $\left.\left./ \mathrm{C}_{\mathrm{Sum}}\right)=.47\right)$ suggest that the WST-1 macrophage assay provides a valid assessment of superoxide anion production by HMDM - especially when considering 
that current production in the fuel cell only partially originates from superoxide anions produced by HMDM, while the level of WST-1 reduction is almost completely based on HMDM superoxide anion release.

There are several potential implications for the implementation of the WST-1 macrophage assay. Thus far in the study of psychosomatic or psychobiological research, there has been a lack not only of methods for investigating microbicidal potential of human macrophages but also of literature regarding associations to longer-lasting psychological states or measures. However, elucidating the relationship between psychological factors and microbicidal potential of macrophages may contribute to a better understanding of the biological mechanisms linking psychological risk factors with adverse health consequences (e.g. increased susceptibility to infectious diseases, impaired wound healing, or coronary heart disease) where activated macrophages and thus peripheral immune cell activity play a major role (13, 27-29). Moreover, several studies have suggested altered inflammatory activity and disturbed neuroendocrine-immune interaction in depression. Notwithstanding the fact that the clinical significance of the observed association between depressive symptoms and macrophage activity in our study remains unclear, it might be worthwhile to further investigate the role of macrophage activity in the interface between inflammation, neuroendocrine-immune interaction, and depression. The WST-1 macrophage assay may facilitate future research in this field.

Based on our implementation and validation data, we recommend the following for the use of the WST-1 macrophage assay: First, in order to investigate effects of potentially influencing factors on basal activity of M1 macrophages blood samples should be taken by means of short-term cannula insertion. This is because our data indicate that an indwelling venous catheter at least if inserted for a longer period of time) seems to function as an open wound and to pre-activate circulating monocytes as precursors of later M1 macrophages in vivo. 
Second, although our data provide first indications that both total and cell-number corrected WST-1 reduction provide similar results in terms of associations with psychological factors we abstain from recommending to interpret total WST-1 reduction as an indicator of microbicidal potential per macrophage. Third, our data show acceptable inter-assay variability which suggests that single measurements of WST-1 reduction provide acceptable results. Nevertheless it might be methodologically stronger to perform the assay in duplicates.

The present study has several strengths. First, it is the first study of tissue-based immune cell activity for use in psychosomatic research. Second, in comparison with other immunological methods or cell functionality assays, the in vitro method implemented in this study requires no expensive laboratory equipment or reagents, nor does it involve complicated procedures. Thus, we introduce a method that is also performable in smaller or less well equipped laboratories. Third, we implemented and validated the WST-1 macrophage assay for two commonly used blood sampling procedures, i.e. short-term cannula insertion and long-term catheter insertion, and in both women and men. The present study also has limitations. First, we did not systematically examine the association of macrophage microbicidal potential or ROS production with macrophage microbicidal activity. Although the important role of ROS in microbicidal activity of human mononuclear phagocytes is well established (6), the relative importance of ROS in the microbicidal activity of mononuclear phagocytes varies depending on the target pathogen (30-33). Future research should examine the association of macrophage microbicidal potential with macrophage microbicidal activity. Second, we only implemented the macrophage assay using WST-1 reduction as detection method for macrophage superoxide anion production but not using other methods for superoxide anion detection, such as chemiluminescence- or flow cytometry-based methods. Further studies are needed to address this. Third, although our study provides first indications that depressive symptom severity and chronic stress affect macrophage microbicidal potential, we did not examine 
these associations systematically. Large-scale studies or studies involving depressive patients are needed to systematically address this (e.g. by comparing macrophage microbicidal potential between psychiatric patients and controls). A final limitation refers to the fact that no exclusion criteria were applied in the cannula group. While this procedure results in a high amount of generalizability on the one hand, we cannot rule out that results obtained from the cannula group or differential results between cannula vs. catheter group are biased by gender differences or potential unknown confounders on the other hand.

In summary, we present a successful in vitro method to determine peripheral immune cell activity. This can be accomplished by investigation of macrophage activation using a valid procedure of determining the NADPH oxidase-mediated microbicidal potential of ex vivo isolated HMDM. The present results indicate that this method is of interest to psychosomatic or psychobiological research as it allows to test for associations of psychological factors with peripheral immune cell activity. Additional research is needed to test the effects of acute mental stress responses and psychological constructs other than chronic stress or depressive symptoms. Future studies may also elucidate the effects of different assay modulation procedures such as variations in concentrations of stimulating agents and how such methods affect the observed associations between psychological factors and immune activation.

\section{$\underline{5 \text { Acknowledgement }}$}

The authors are grateful to cand. b.sc. Andrina Foppa and cand. m.sc. Mark Hauser for laboratory assistance.

\section{$\underline{6 \text { List of Supplemental Digital Content }}$}


Supplemental Digital Content 1. Determination of assay reliability. doc

Supplemental Digital Content 2. Cytochrome c assay. doc

Supplemental Digital Content 3. Operation of the biofuel cell.doc

Supplemental Digital Content 4. Cell-counting method.doc 


\section{$\underline{6 \text { References }}$}

1. Ader R, editor. Psychoneuroimmunolgy 4ed. Amsterdam: Elsevier Academic Press.; 2006.

2. Rabin B. Stress, immune function, and health: the connection. New York: Wiley-Liss.; 1999.

3. Taylor PR, Martinez-Pomares L, Stacey M, Lin HH, Brown GD, Gordon S. Macrophage receptors and immune recognition. Annu Rev Immunol. 2005;23:901-44.

4. Kuebler U, Wirtz PH, Sakai M, Stemmer A, Ehlert U. Acute stress reduces woundinduced activation of microbicidal potential of ex vivo isolated human monocyte-derived macrophages. PLoS One. 2013;8:e55875.

5. Schroder K, Hertzog PJ, Ravasi T, Hume DA. Interferon-gamma: an overview of signals, mechanisms and functions. J Leukoc Biol. 2004;75:163-89.

6. de Oliveira-Junior EB, Bustamante J, Newburger PE, Condino-Neto A. The human NADPH oxidase: primary and secondary defects impairing the respiratory burst function and the microbicidal ability of phagocytes. Scand J Immunol. 2011;73:420-7.

7. El-Benna J, Dang PM, Gougerot-Pocidalo MA, Elbim C. Phagocyte NADPH oxidase: a multicomponent enzyme essential for host defenses. Arch Immunol Ther Exp (Warsz). 2005;53:199-206.

8. Sakai M, Vonderheit A, Wei X, Kuttel C, Stemmer A. A novel biofuel cell harvesting energy from activated human macrophages. Biosens Bioelectron. 2009;25:68-75.

9. Martinez FO, Sica A, Mantovani A, Locati M. Macrophage activation and polarization. Front Biosci. 2008;13:453-61.

10. Dale DC, Boxer L, Liles WC. The phagocytes: neutrophils and monocytes. Blood. 2008;112:935-45. 
11. Cathcart MK. Regulation of superoxide anion production by NADPH oxidase in monocytes/macrophages: contributions to atherosclerosis. Arterioscler Thromb Vasc Biol. 2004;24:23-8.

12. Tan AS, Berridge MV. Superoxide produced by activated neutrophils efficiently reduces the tetrazolium salt, WST-1 to produce a soluble formazan: a simple colorimetric assay for measuring respiratory burst activation and for screening anti-inflammatory agents. $\mathrm{J}$ Immunol Methods. 2000;238:59-68.

13. Mahdavian Delavary B, van der Veer WM, van Egmond M, Niessen FB, Beelen RH. Macrophages in skin injury and repair. Immunobiology. 2011;216:753-62.

14. Geng YJ, Hansson GK. Interferon-gamma inhibits scavenger receptor expression and foam cell formation in human monocyte-derived macrophages. J Clin Invest. 1992;89:132230.

15. Pawlowski NA, Abraham EL, Pontier S, Scott WA, Cohn ZA. Human monocyteendothelial cell interaction in vitro. Proc Natl Acad Sci U S A. 1985;82:8208-12.

16. Pawlowski NA, Kaplan G, Hamill AL, Cohn ZA, Scott WA. Arachidonic acid metabolism by human monocytes. Studies with platelet-depleted cultures. J Exp Med. 1983;158:393-412.

17. Mach F, Schonbeck U, Bonnefoy JY, Pober JS, Libby P. Activation of monocyte/macrophage functions related to acute atheroma complication by ligation of CD40: induction of collagenase, stromelysin, and tissue factor. Circulation. 1997;96:396-9.

18. Selvan RS, Zhou LJ, Krangel MS. Regulation of I-309 gene expression in human monocytes by endogenous interleukin-1. Eur J Immunol. 1997;27:687-94.

19. Pelegrin P, Surprenant A. Dynamics of macrophage polarization reveal new mechanism to inhibit IL-1beta release through pyrophosphates. EMBO J. 2009;28:2114-27. 
20. Ma J, Liu L, Che G, Yu N, Dai F, You Z. The M1 form of tumor-associated macrophages in non-small cell lung cancer is positively associated with survival time. BMC cancer. 2010;10:112.

21. Babior BM, Kipnes RS, Curnutte JT. Biological defense mechanisms. The production by leukocytes of superoxide, a potential bactericidal agent. J Clin Invest. 1973;52:741-4.

22. Hautzinger M, Bailer M, Worall H, Keller F. Beck-Depressions-Inventar (BDI). Bern: Hans-Huber; 1994.

23. Schulz P, Schlotz W, Becker P. Trierer Inventar zum chronischen Stress. Göttingen: Hogrefe; 2004.

24. Dorshkind K, Montecino-Rodriguez E, Signer RA. The ageing immune system: is it ever too old to become young again? Nat Rev Immunol. 2009;9:57-62.

25. Wirtz PH, Ehlert U, Emini L, Suter T. Higher body mass index (BMI) is associated with reduced glucocorticoid inhibition of inflammatory cytokine production following acute psychosocial stress in men. Psychoneuroendocrinology. 2008;33:1102-10.

26. Schuurs AH, Verheul HA. Effects of gender and sex steroids on the immune response. Journal of steroid biochemistry. [Review]. 1990;35:157-72.

27. Ross R. Atherosclerosis--an inflammatory disease. N Engl J Med. 1999;340:115-26.

28. Rozanski A, Blumenthal JA, Davidson KW, Saab PG, Kubzansky L. The epidemiology, pathophysiology, and management of psychosocial risk factors in cardiac practice: the emerging field of behavioral cardiology. J Am Coll Cardiol. 2005;45:637-51.

29. Gouin JP, Kiecolt-Glaser JK. The impact of psychological stress on wound healing: methods and mechanisms. Immunol Allergy Clin North Am. 2011;31:81-93.

30. Murray HW, Rubin BY, Carriero SM, Harris AM, Jaffee EA. Human mononuclear phagocyte antiprotozoal mechanisms: oxygen-dependent vs oxygen-independent activity against intracellular Toxoplasma gondii. J Immunol. 1985;134:1982-8. 
31. Cohen MS, Isturiz RE, Malech HL, Root RK, Wilfert CM, Gutman L, Buckley RH. Fungal infection in chronic granulomatous disease. The importance of the phagocyte in defense against fungi. Am J Med. 1981;71:59-66.

32. Vazquez-Torres A, Balish E. Macrophages in resistance to candidiasis. Microbiol Mol Biol Rev. 1997;61:170-92.

33. Levitz SM, Diamond RD. A rapid colorimetric assay of fungal viability with the tetrazolium salt MTT. J Infect Dis. 1985;152:938-45. 
Table 1

Table 1. Group characteristics of participants of study sample 2 and study sample 3

\begin{tabular}{|l|c|c|c|}
\hline & $\begin{array}{c}\text { Study sample 2 } \\
\text { (cannula group) }\end{array}$ & $\begin{array}{c}\text { Study sample 3 } \\
\text { (catheter group) }\end{array}$ & \\
\hline Gender (n; men/ & $n=40$ & $n=24$ & \\
women) & $16 / 24$ & $24 / 0$ & .46 \\
\hline Age (yr) & & & .15 \\
\hline BMI (kg/m ${ }^{2}$ ) & $24.1 \pm 0.6$ & $25.8 \pm 1.0$ & $<.001$ \\
\hline Total WST-1 (OD) & $0.070 \pm 0.007$ & $0.283 \pm 0.021$ & \\
\hline Corrected WST-1 & $0.040 \pm 0.008$ & & -022 \\
(OD) & & & \\
\hline Chronic stress (CSSS) & $22.9 \pm 2.0$ & & \\
\hline Depressive symptom & $6.9 \pm 1.1$ & & \\
\hline severity (BDI) & & & \\
\hline
\end{tabular}

Values are means \pm SEM. BMI = body mass index; OD = optical density; CSSS = chronic stress screening scale; BDI = beck depression inventory; $\mathrm{n}$ = number of participants. 


\section{Figure legends}

Figure 1. Pilot data. Values are given as mean \pm SEM. Figure $1 \mathrm{~A}$ depicts WST-1 reduction by ex vivo isolated human monocyte-derived macrophages after differential stimulation in a cannula and a catheter group. From the left: Non-stimulated cells as negative control; activation with $50 \mathrm{nM}$ PMA; differentiation with $300 \mathrm{ng} / \mathrm{ml} \mathrm{LPS,} 20 \mathrm{ng} / \mathrm{ml}$ IFN- $\gamma$, and 20 ng/ml TNF- $\alpha$; differentiation and activation stimuli (300 ng/ml LPS, 20 ng/ml IFN- $\gamma, 20$ ng/ml TNF- $\alpha, 50$ nM PMA). Across all measurements the combined differentiation and activation stimulation condition differed significantly from all of the other conditions ( $p$ 's $\leq$ .038). Figure 1B depicts PMA-induced superoxide anion production by macrophages with WST-1 reduction scores plotted against increasing concentrations of peripheral blood mononuclear cells (PBMCs).

Figure 2. Validity. The scatter plot depicted in Figure 2A shows reduced cytochrome c and WST-1 reduction scores (cannula group). Figure 2B depicts the maximum current value registered $\left(\mathrm{C}_{\text {Max }}\right)$ and WST-1 reduction scores (catheter group) while the sum of all current values $\left(\mathrm{C}_{\text {Sum }}\right)$ and WST-1 reduction scores (catheter group) is depicted in Figure 2C. 
Figure 1A

A

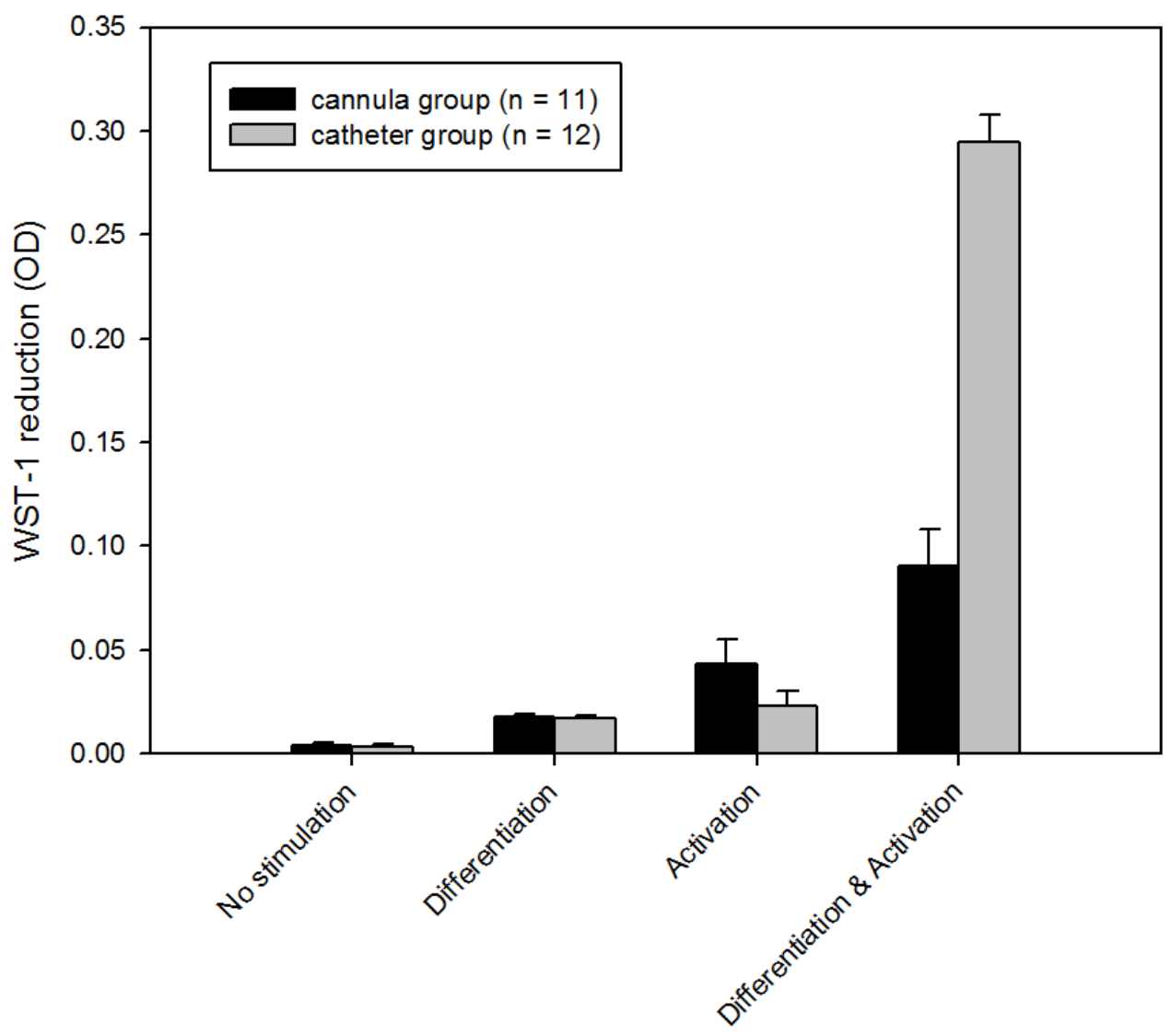


Figure 1B

B

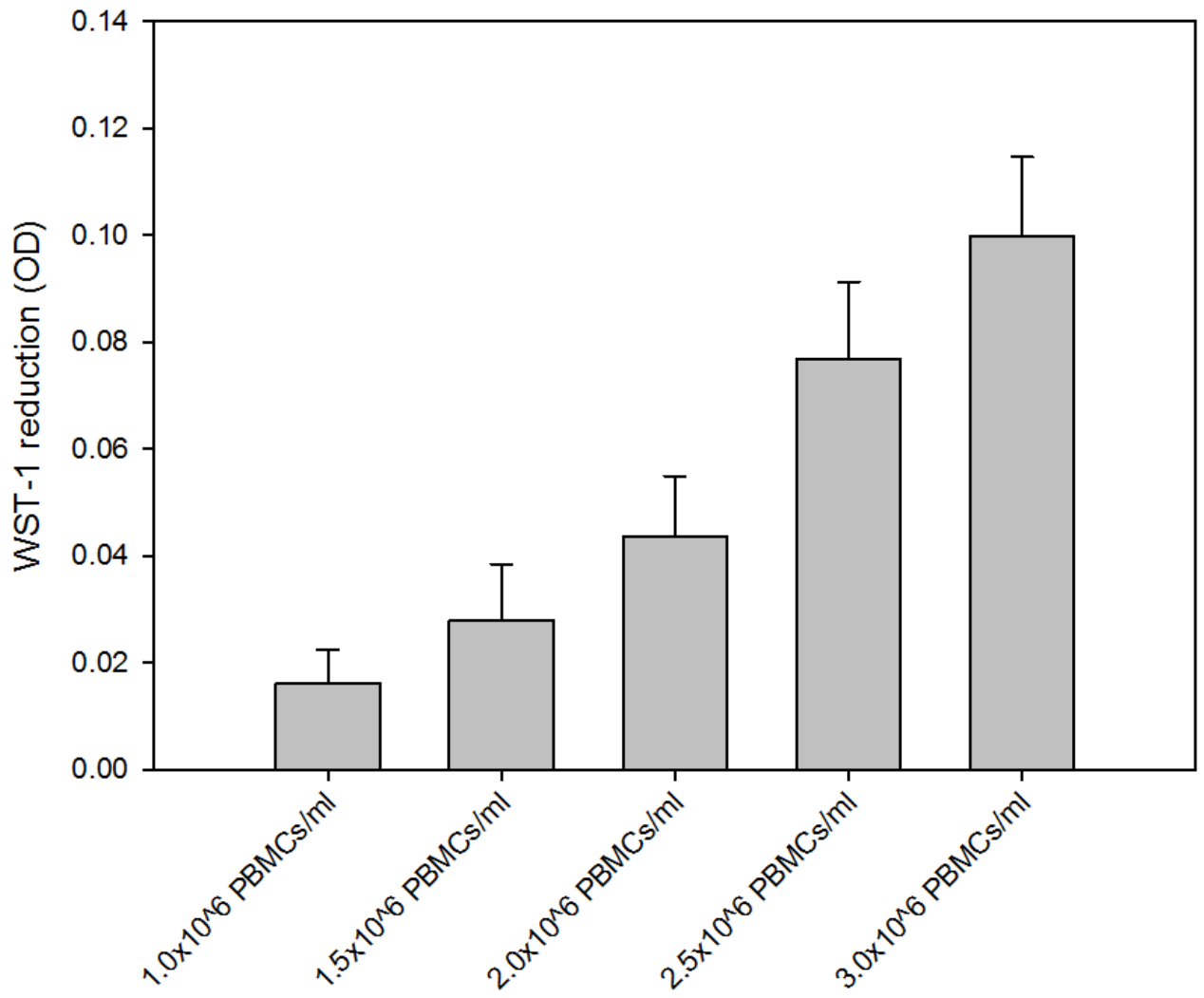


Figure 2A

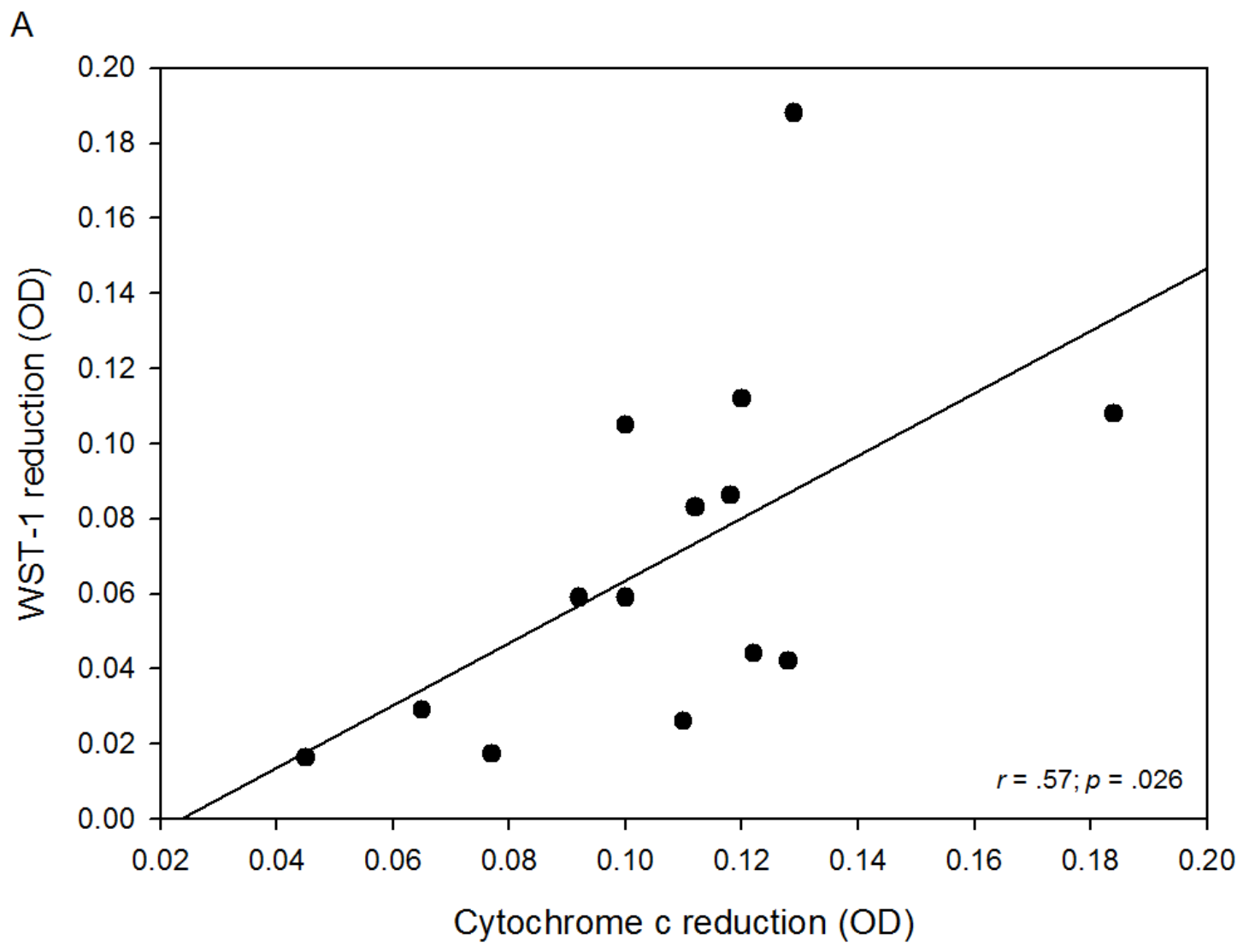


Figure 2B

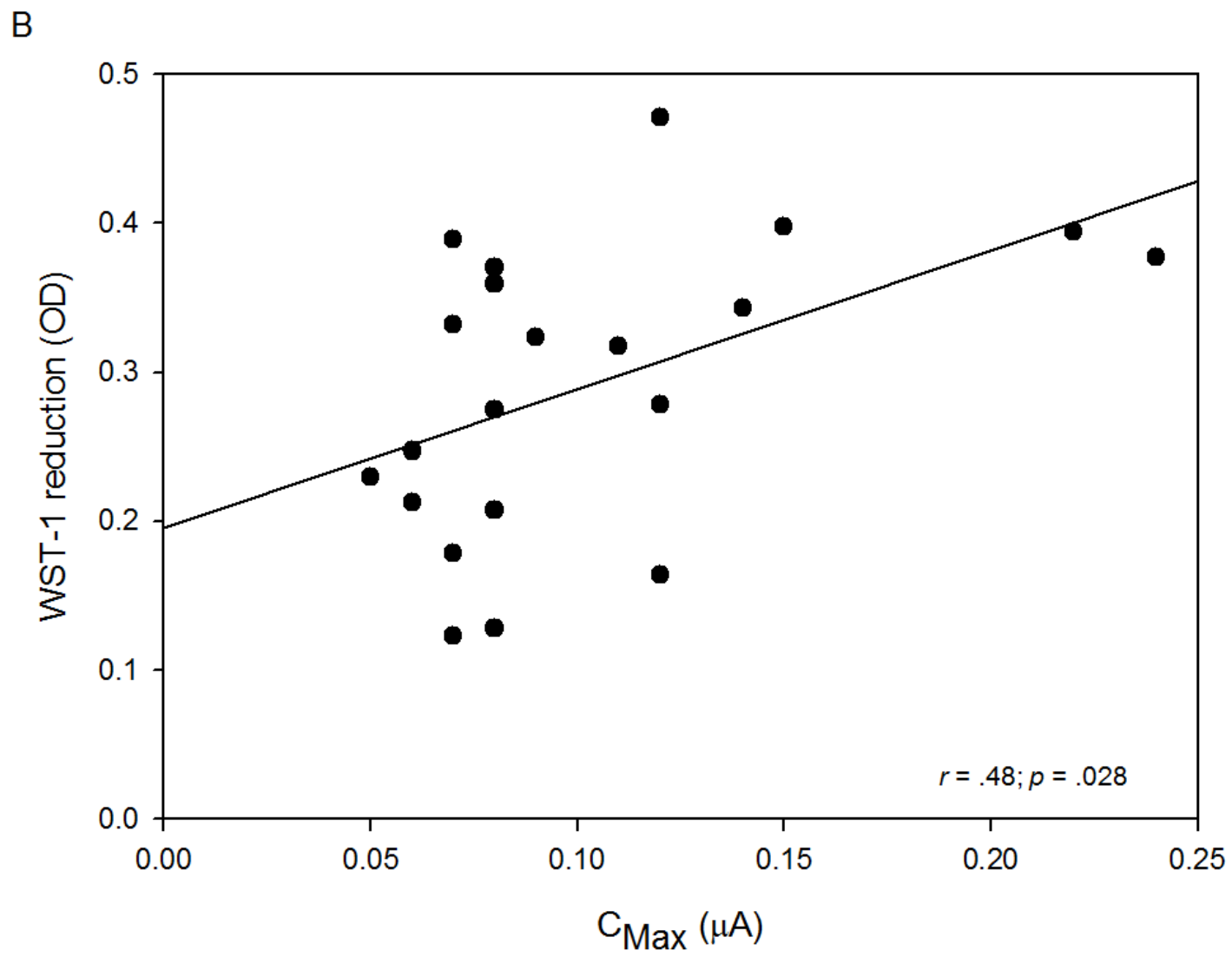


Figure 2C

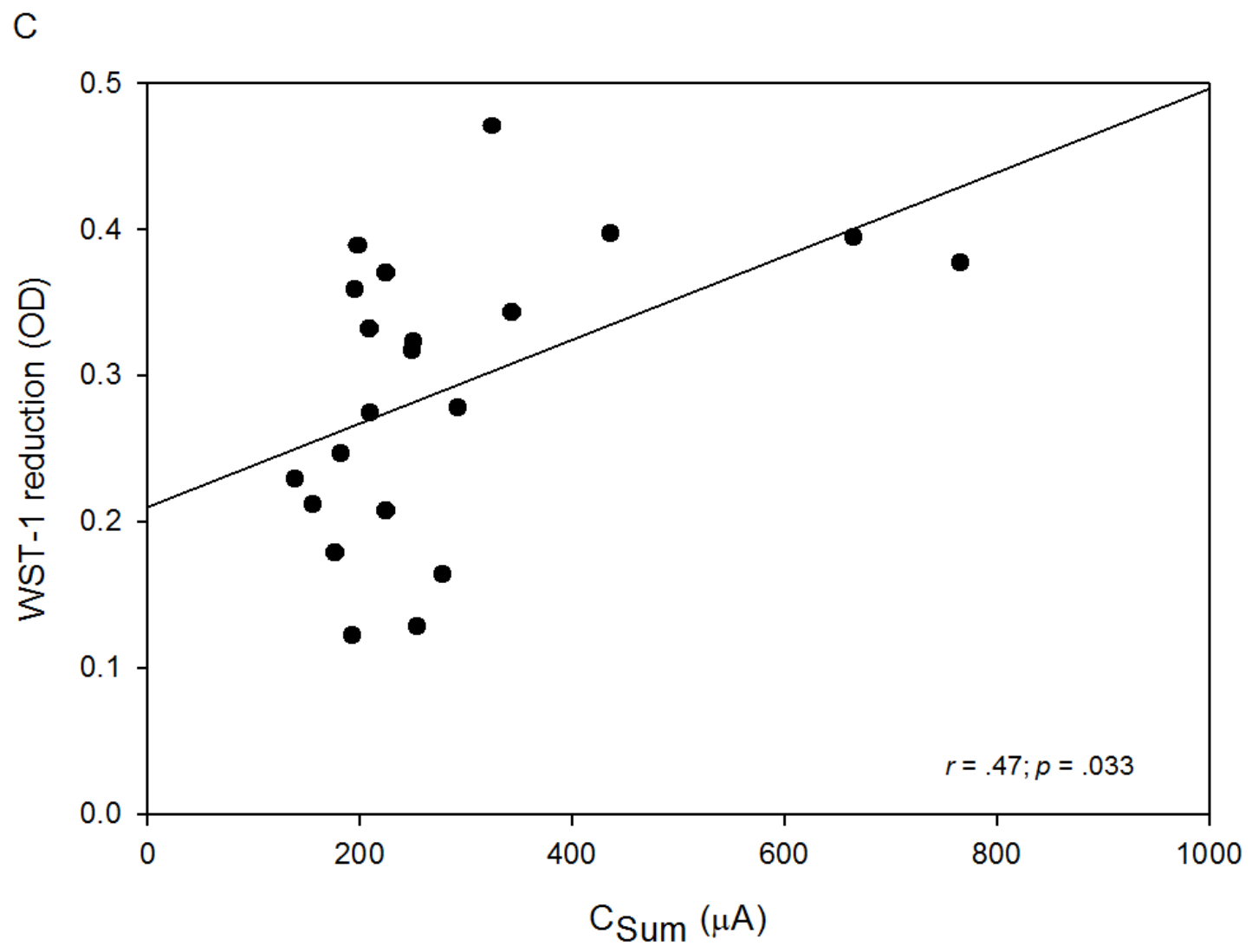

